\title{
ON TWO NEW CHARACTERIZATIONS OF STIELTJES TRANSFORMS FOR DISTRIBUTIONS
}

\section{SUNIL KUMAR SINHA}

\author{
Department of Mathematics \\ Tata College Chaibasa, Singhbhum \\ Bihar-833202, INDIA \\ (Received September 27, 1981)
}

\begin{abstract}
Two new characterizations of the Stieltjes transform for distribution are developed, using two transformations on the space of distributions viz., diation $U_{n}$ and exponential shifts $T^{-P}$. The standard theorem on analyticity, uniqueness and invertibility of the Stieltjes transform are proved, using the new characterization as the definition of the Stieltjes transform.
\end{abstract}

KEY WORDS AND PHRASES. Stieltjes transform of distribution, iterated Laplace transform, diicitiuns, exponertial shifts.

1980 AMS SUBJECT CLASSIFICATION CODE. $46 F 12$.

1. INTRODUCTION.

Widder ([1], p. 325) introduced the Stieltjes transform as an iteration of the Laplace transform as follows:

where

$$
f(x)=\int_{0}^{\infty} e^{-x t} \phi(t) d t
$$

so that

$$
\begin{aligned}
& \phi(x)=\int_{0}^{\infty} e^{-x t} \psi(t) d t, \\
& f(x)=\int_{0}^{\infty} \frac{\psi(t) d t}{(x+t)}
\end{aligned}
$$

More generally, (1.3) can be replaced by Stieltjes integral

$$
f(x)=\int_{0}^{\infty} \frac{d \alpha(t)}{(x+t)}
$$

The integral (1.4) was originally considered by Stieltjes[2]. Various generalizations of Stieltjes transform have been given by various authors viz., Widder[3], Pollard[4], Sumner[5], Mishra[6], Pathak([7]-[8]), Rao[9], Varma[10], Arya([11]-[15]), Ghosh([16][18]), Boas \& Widder[19], and Dube[20].

The present paper is concerned with two new characterizations of Stieltjes transformation for distributions by the help of dilation $U_{n}$ and exponential shifts $\mathrm{T}^{-\mathrm{p}}$ introduced earlier by Gesztelyi[21]. It is interesting to note here that Gestzelyi considered two transformations viz., dilation $U_{n}$ and exponential shifts $T^{-p}$ which are defined for ordinary functions $f$, complex number $p$, and positive integer $n$ 
by:

$$
\begin{aligned}
& u_{n} f(t)=n f(n t) \\
& T^{-p} f(t)=e^{-p t} f(t)
\end{aligned}
$$

Gesztelyi proves that if $f$ is a function which has a Laplace transform at $p$, then the sequence function $\left\{u_{n} T^{-P} f(t)\right\}$ converges (in Mikusinski sense) as $n \rightarrow \infty$ to the classical Laplace transform of $f$ at $p$. He then defines the Laplace transform of a Mikusinski operator $\mathrm{x}$ as the limit (whenever it exist in the sense of Mikusinskiconvergence) of the sequence $\left\{u_{n} T^{-p} x\right\}$, and shows that his definition generalizes the previous formulation of the Laplace transform of Mikusinski operators of G. Doetsch [23] and V. A. Ditkin ([24]-[25]). Price[26] defined the Laplace transformation of a distribution $f$ using sequences of the form $\left\{u_{n} T^{-p} f\right\}$ and shows that the new definition is equivalent to Schwartz's extension of the transform to distributions. He also introduced spaces $B$ and $B_{0}$ and their duals $B^{\prime}$ and $B_{O}^{\prime}$ and shows that each distribution $f$ in $B_{O}^{\prime}$ has a unique extension $f$ in $B^{\prime}$. He also shows that the sequence $\left\{U_{j} f\right\}$ converges to $\langle f, l\rangle \delta$ whenever $f$ is in $B_{o}^{\prime} \cdot$ Recently, working on the same lines the present author has given two new characterizations of the Weierstrass, Mel1in, Hankel and K-transform for distributions([28], [29], [30]\& [31]). 2. TWO NEW CHARACTERIZATIONS OF THE STIELTJES TRANSFORMS.

In the present section we give two new characterizations of stieltjes transforms for one-dimensional distributions.

We will say that a distribution $f$ is Stieltjes transformable if there is an open interval $(\alpha, \beta)$ such that whenever $p=\sigma+i \tau$ is a complex number with real part in $(\alpha, \beta) ; T^{-P_{f}}$ is a distribution in $B_{O}^{\prime}$, where $B_{0}^{\prime}$ is the dual space of $B_{0}$, a subspsace of ${ }^{-x}$, as defined in [27].

If $(\alpha, \beta)$ is the largest such open interval then the set $\Omega=\{p: \operatorname{Re} p \varepsilon(\alpha, \beta)\}$ is called the domain of definition of the Stieltjes transform for $f$.

If $f$ is a Stieltjes transformable distribution, where transform has domain definition $\Omega$, then for $p \in \Omega$, we define the Stieltjes transform $s[f](p)$ of $f$ at $p$ by

$$
S[f](p)=\frac{1}{\phi(0)} \lim _{j \rightarrow \infty}\left\langle u_{j} T^{-p} f, \phi\right\rangle,
$$

where $f(t)=S[\psi](p), 0 \leq t \leq R$ for every positive $R, \phi$ is a test function in $\not$ with $\phi(0) \neq 0$ and $\psi$ is another distribution.

\{NOTE: For the existence of limit in (2.1) and its meaning one could focus his attention on Theorem 3.1, p. 24 [26] or [27].\}

Thus we have another characterization also as

$$
S[f\rceil(p)=\left\langle T^{-p} f, 1\right\rangle
$$

where $f(t)=S[\psi](p), 0 \leq t \leq R$ for every positive $R$ and $\psi$ is another distribution. 
From (2.2) we see that $S[f](p)$ is a complex valued function of the complex variable $p$ with domain $\Omega$. In fact, the mapping $S$ is linear. For, if $f$ and $g$ are distributions that are transformable at $p$ and $a$ and $b$ are complex numbers then $(a f+b g)$ is Stieltjes transformable at $p$ and

$$
\begin{aligned}
& \mathrm{s}[\mathrm{af}+\mathrm{bg}](\mathrm{p})=\left\langle\mathrm{T}^{-\mathrm{p}}[\mathrm{af}+\mathrm{bg}], 1\right\rangle
\end{aligned}
$$

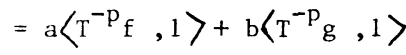

$$
\begin{aligned}
& =a S[f](p)+b S[g](p) \text {. }
\end{aligned}
$$

THEOREM 2.1. If $\mathrm{f}$ is a distribution that is Stieltjes transformable in $\Omega$, then $\mathrm{S}: \mathrm{fl}(\mathrm{p})$ is analytic function of $\mathrm{p}$ in $\Omega$ and

$$
\frac{d}{d p} S[f](p)=S[-t f(t)](p) \text {. }
$$

PROOF. The proof is analogous to that for Laplace transformation as given in [26] $|27|$.

3. TREATMENT OF THE CONVOLUTION OF TWO DISTRIBUTIONS.

Much of the usefulness of the Stieltjes transform is a result of the way it treats the convolution of two distributions. We give here this important property of the transformation by the following theorem:

THEOREM 3.1. If $\mathrm{f}$ and $\mathrm{g}$ are Stieltjes transformable distributions such that the domain of their respective transforms have intersection $\Omega$, then $f * g$ is Stieltjes transformable in $\Omega$ and for every $p$ in $\Omega \quad S[f * g](p)=S[f](p) S[g](p)$. PROOF. For $p$ in $s, T^{-P_{f}}$ and $T^{-p} g$ are both in $B_{O}^{\prime}$. Therefore, $f^{*} g$ is Streltjes transformable at $\mathrm{p}$, and from (2.2) and the definition of convolution we get

$$
\begin{aligned}
& S[f * g](p)=\left\langle T^{-p}(f * g), 1\right\rangle
\end{aligned}
$$

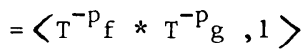

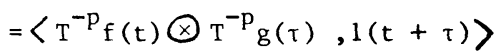

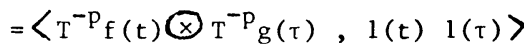

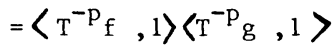

$$
\begin{aligned}
& =S[f](p) S[g](p) \text {. }
\end{aligned}
$$$$
\text { Q.E.D. }
$$

4. INVERSION AND UNIQUENESS THEOREMS FOR THE STIELTJES TRANSFORM.

No theory of Stieltjes transform would be useful without the inverison and uniqueness theorems. We give Theorem 4.1 which includes both inversion and uniqueness theorems as its corollary.

In what follows we will have as independent variables at various times the real variable $t$ and real and imaginary parts of complex variable $p$. For reason we will sometime indicate the particular independent variable for the space or an operation by a subscript $\tau$, e.g., $\left\langle f(\tau), e^{-i \omega t}\right\rangle_{\tau}$, where $f(\tau)$ is in $B_{0}^{\prime}$ and $\omega$ is a parameter. 
THEOREM 4.1. If $\mathrm{f}$ is a distribution in $\mathrm{B}_{\mathrm{O}}^{\prime}$, then

$$
f(t)=-\frac{1}{2 \pi} \lim _{r \rightarrow \infty} \int_{-r}^{r} e^{i \omega t}\left\langle f(\tau), e^{-i \omega \tau}\right\rangle{ }_{\tau} d \omega,
$$

where the 1 imit is taken in $\overbrace{t}^{\prime}$.

PROOF. The proof is same as that of Laplace transform as given in [27].

COROLLARY 4.1 (a): (Inversion theorem).

If $f$ is Stieltjes transformable in $\Omega=\{p: \alpha<\operatorname{Re} p<\beta\}$. Then, as long as $\alpha<\sigma<\beta$

where the limit is taken in " $z_{t}^{\prime}$.

COROLLARY 4.1(b): (Uniqueness theorem).

If $f$ and $g$ are Stieltjes transformable distributions such that $S[f](p)=$ $\mathrm{S}[\mathrm{g}](\mathrm{p})$ on some vertical line in the common domain of transforms of $\mathrm{f}$ and $g$, then $\mathrm{f}=\mathrm{g}$ as distributions.

\section{REFENCES}

1. WIDDER, D. V. The Laplace Transform, Princeton University Press, 1941, latest ed. 1963.

2. WIDDER, D. V. The Stieltjes Transform, Trans. Amer. Math. Soc., 43 (1938) 7-60.

3. STIEltJES, T. J. Recherches sur les fractions continues. Annal de le faculte' des sciences de Toulouse, Vol. 8, pp. 1-122(1894).

4. POLlARD, H. Studies on the Stieltjes-transform, Ph.D. thesis, Harvard Univ., 1942.

5. SUMNER, D. B. A convolution transform Admitting an inversion Formula of Integro Differential Type, Canad. J. Maths, 1953, 114-117.

6. MISRA, B. P. Some Abelian theorems for distributional Stieltjes transformation, Jour. of Math. Anal. and Appl. 39 (1972), 590-599.

7. PATHAK, R. S. A distributional generalized Stieltjes transformation, Proc. Edin. Math. 20 (1976), 15-22.

8. PATHAK, R.S. A representational theorem for a class of Stieltjes transformable functions, Jour. Ind. Math. Soc. 38(1974), 339-344.

9. RAO, G. L. N. Abelian theorems for a distributional generalized Stieltjes transforms, Revistade la Real academia de ciencias extacts, fisicas Y naturales Madrid, Tomo L XXO cuaderno 1, pp 97-108, 1976.

10. VARMA, R.S. On a generalization of Laplace integral, Proc. Nat. Acad. Sc. 20A (1951), 209-216.

11. ARYA, S. C. Abelian theorem for generalized Stieltjes Transform, Bull. U.M.I. (3) $13,1958,497-504$.

12. ARYA, S. C. Convergence theorems and asymptotic properties of generalized Stieltjes transform, Jour. Ind. Math. Soc., 22, (1958), 119-135.

13. ARYA, S. C. Inversion theorems for a generalized Stieltjes transform, Rivista di. Math. (Univ. di Parma) 9 (1958), 139-148.

14. ARYA, S. C. A real inversion theorem for a generalized Stieltjes transform, Coll. Math. 10, (1958), 69-79.

15. ARYA, S. C. A complex inversion formula for a generalized Stieltjes transform, Agra Univ. Jour. Of Res.9, (1960), 233-242. 
16. GHOSH, J. D. Studies of generalized Stieltjes transforms and Generalized Hankel transforms of distributions, Ph.D. thesis, Ranchi Univ.,1974.

17. GHOSH, J. D. A real inversion formula for generalized Stieltjes transform of generalized functions, Aligarh Bul1. Math. 3(1973).

18. GHOSH, J. D. On a generalized Stieltjes transform of a class of generalized functions, Bul1. Cal. Math. Soc., 67(1975),75-85.

19. BOAS, R. P. and WIDDER, D. V. The Iterated Stieltjes transform, Trans. Amer. Math. Soc. , 45 (1939), 1-72.

20. DUBE, L. S. An inversion of the $\mathrm{S}_{2}$-transform for generalized functions, Pac. Jour. Math., 61(1975), 383-390.

21. GESZTILYI, E. Uber Lineare operator transformationen,Pub1. Math. (Debrecen), 14, 169-206.

22. MIKUSINSKI, J. Operational Calculus, Pergman Press, N.Y.,1959.

23. DOETSCH, G. Handbunch der Laplace-Transformation, Band I., Birkhouser verlag, Basel, 1950.

24. DITKIN, V. A. On the theory of the operational calculus, Dokl.Akad.Nauk.123, (1958), 395-396.

25. DITKIN, V. A. and PRUDNIKOV, A. P. Integral Transforms and operational calculus, Pergaman Press,N. Y., 1965.

26. PRICE, D. B. On the laplace transform for distributions, SIAM 3. Math. Ana1. 6 (1975) 49-80.

27. PRICE, D. B. On the Laplace transform for distributions, Ph.D. thesis, North Carolina State University at Kaleigh,1973(pp.1-79).

28. SINHA, S. K. On two new characterizations of the Weierstrass transform for Distributinns-to appear.

29. SINHA, S. K. On two new characterizations of the K-transform for Distributionsto appear.

30. SINHA, S. K. On two new characterizations of the Hankel transform for Distributions-to appear.

31. SINHA, S. K. On two new characterizations of the Mellin transform for Distributions-to appear. 


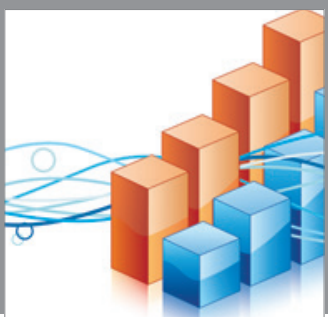

Advances in

Operations Research

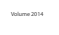

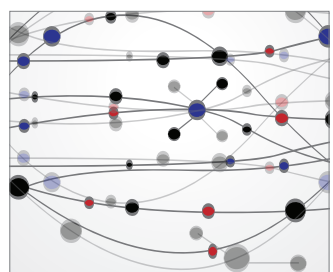

\section{The Scientific} World Journal
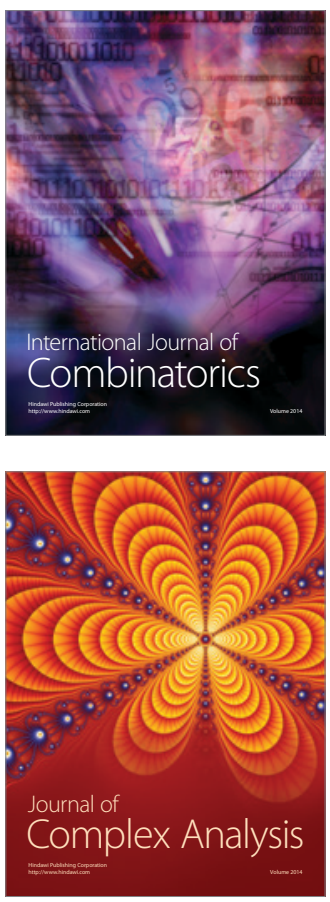

International Journal of

Mathematics and

Mathematical

Sciences
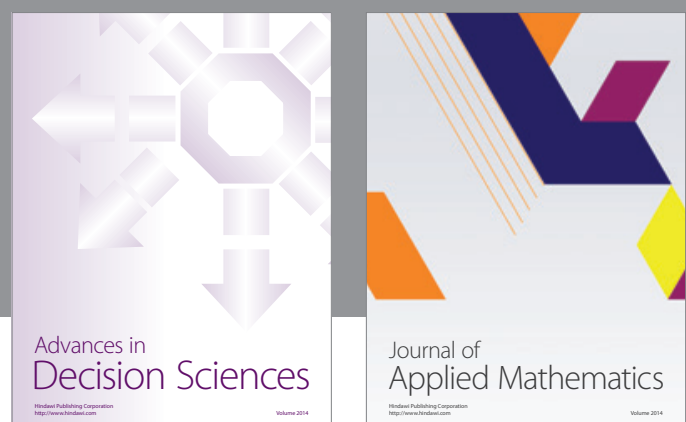

Journal of

Applied Mathematics
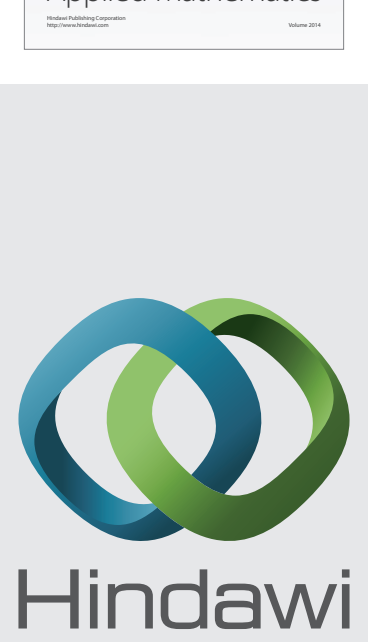

Submit your manuscripts at http://www.hindawi.com
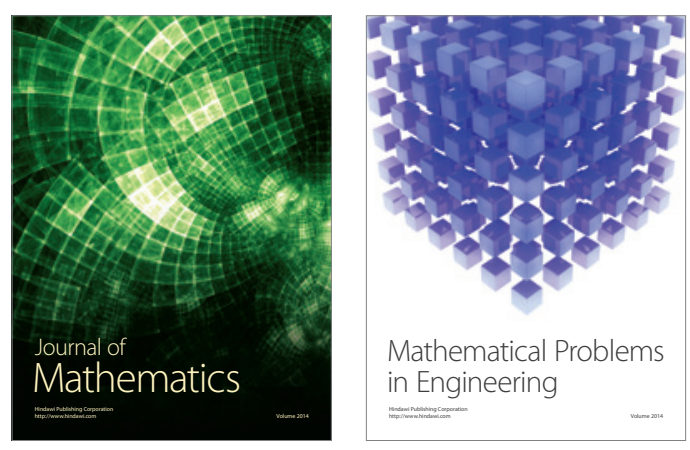

Mathematical Problems in Engineering
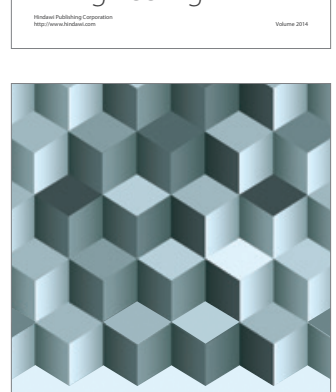

Journal of

Function Spaces
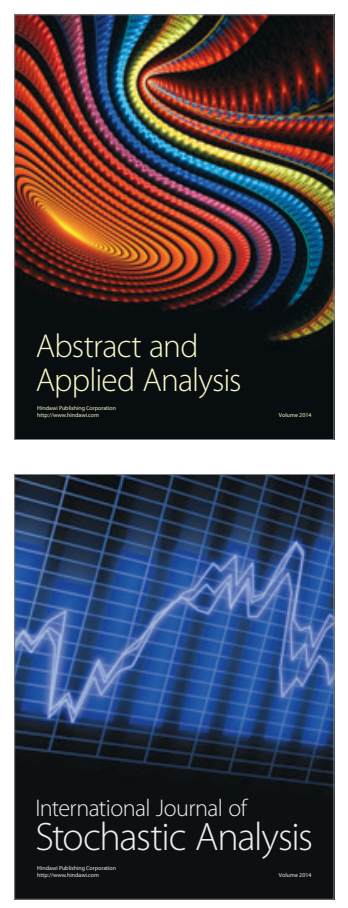

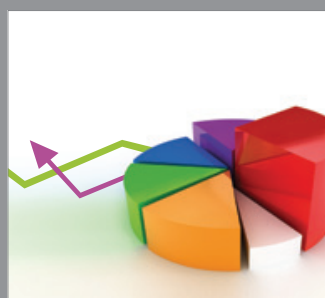

ournal of

Probability and Statistics

Promensencen
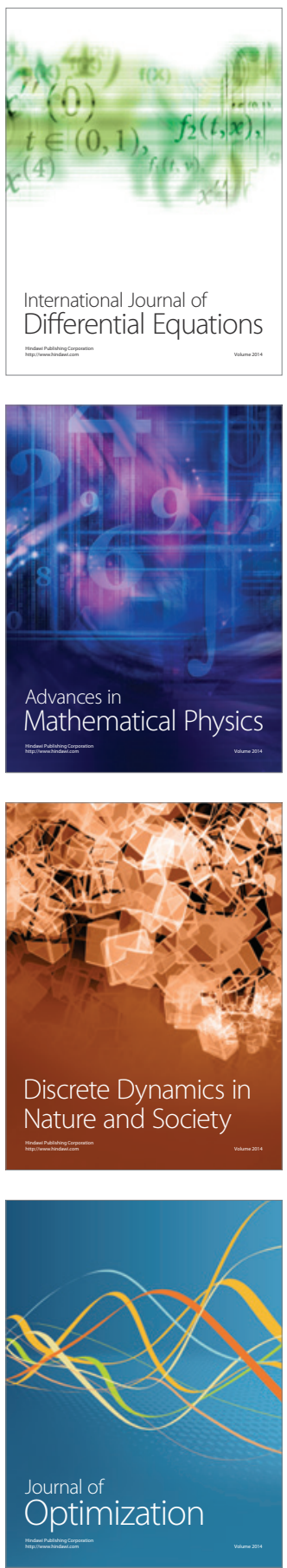